\title{
Do treatment patterns alter beliefs cancer patients hold regarding oral oncolytic agents?
}

\author{
Alla Sikorskii $^{1}$ | Charles W. Given ${ }^{1}$ | Barbara A. Given ${ }^{1}$ (i) | Eric Vachon ${ }^{1}$ | \\ Victoria Marshall $^{1}$ | John C. Krauss ${ }^{2}$ | Asish Banik ${ }^{1}$ | Atreyee Majumder ${ }^{1}$
}

${ }^{1}$ Michigan State University, East Lansing, MI, USA

${ }^{2}$ University of Michigan, Ann Arbor, MI, USA Correspondence

Barbara A. Given, PhD, RN, Michigan State University, College of Nursing C383 Bott Building, East Lansing, MI 48824, USA.

Email: bgiven@msu.edu

Funding information

National Cancer Institute, Grant/Award Numbers: 1R01CA162401-01A1, 1R01CA16240101A1. 2013-2017

\begin{abstract}
Objective: Cancer patients, particularly those prescribed with oral oncolytic medications, face treatment side effects and temporary and permanent stoppages of treatment. This research examines how events during treatment affect patients' beliefs regarding oral oncolytic medications.

Methods: A total of 272 cancer patients initiating 1 of 28 oral oncolytic agents were followed for 12 weeks. Assessments of Beliefs About Medications Questionnaire, symptoms, physical function, and depression measures were performed during telephone interviews at intake (medication start) and 4, 8, and 12 weeks. Electronic medical record audits identified dates of temporary and permanent medication stoppages. Linear mixed-effects models were used for longitudinal analyses of the Beliefs About Medications Questionnaire scores in relation to patient characteristics, symptom severity, and medication stoppages.
\end{abstract}

Results: Over the initial 12 weeks, beliefs about the necessity of oral medications have increased, concerns have decreased, and interference of medications with daily lives has increased. Permanent stoppage of a medication predicted significant declines in beliefs about its necessity over time. Male patients, those less educated, those reporting higher symptom severity, and those experiencing temporary stoppages had greater concerns. Interference of medications with daily life was higher for males, increased with higher symptom severity, and differed by drug category.

Conclusions: Patients' beliefs in the necessity of their oral medication were affected only by a permanent drug stoppage. Symptom severity, education, and patient sex affected patients' beliefs about their concerns with their medications and the interference medications posed for their daily lives. Interventions may need to target the distinct dimensions of beliefs during treatment with oral oncolytic agents.

\section{KEYWORDS}

cancer, late stage disease, medication beliefs, medication stoppages, oncology, oral oncolytic medications, symptoms

\section{1 | BACKGROUND}

Patients with advanced cancers for whom earlier lines of treatment have failed are frequently prescribed with oral oncolytic agents including oral cytotoxic drugs, inhibitors directed at cell surface receptors, and those targeted at the tumor microenvironment. ${ }^{1} \mathrm{~A}$ number of features set these medications apart from medications for other chronic conditions. Oral oncolytic medications are typically expensive with a cost of hundreds of United States (US) dollars per dose and, although covered by many health insurances in the US, require out-of-pocket costs that could be in thousands of dollars. These medications may produce severe symptoms and side effects that patients experience at home without frequent contacts with oncology professionals. In response to these symptoms and side effects, oncologists may adjust 
the dose by temporarily stopping the medication or permanent discontinuation of the oral oncolytic agent. This research focuses on examining factors that influence patients' beliefs about oral oncolytic medications as they proceed through the first weeks of treatment.

Horne and colleagues ${ }^{2,3}$ developed measures of patients' beliefs regarding the necessity of their medications and concerns that arise from their impact on functioning and health. The Beliefs about Medicines Questionnaire (BMQ) is grounded in social cognition as expressed through the health belief model and assesses patients' positive outcome expectancy (benefits from treatment) and the accompanying concerns regarding the use of medications and their interference with daily activities. ${ }^{4-6}$ Patients' scores on the necessity and concerns subscales have been found to be associated with their levels of adherence ${ }^{2,7-9}$ as well as with their sex, symptom reports, duration of treatment, number of medications, and depression.,10-17

This research examines how events arising during the course of treatment together with patients' factors alter beliefs regarding the necessity and concerns attributed to their medications. Identifying the effects of these events and patient factors could inform the development and tailoring of interventions to support adherence to and persistence of oral oncolytic treatment.

This report is based on the secondary analysis of data from a recently completed trial of an intervention to improve adherence and symptom management among patients' newly prescribed oral oncolytic medication. ${ }^{18}$ Briefly, adherence was high and did not differ by trial arm, while symptoms were improved in the experimental arm. The important feature of this trial is the timing of data collection beginning with the initiation of the new oral oncolytic agent and through the first 12 weeks. We assess these data to determine how medication beliefs change over these initial 12 weeks and how patient, disease, and treatment factors influence the beliefs over time.

\section{2 | METHODS}

\section{1 | Design and sample}

A total of 272 patients were accrued from 6 national cancer institutedesignated comprehensive cancer centers in the US (Figure 1). The study was approved by the Institutional Review Board (IRB) of Michigan State University (IRB\# 13-076 M) and the IRBs of each cancer center. All patients had had health insurance coverage that was private and/or government-sponsored (Medicaid, Veterans Administration, Medicare for those 65 years of age or older). Patients were identified when first prescribed at least 1 of 28 oral anticancer agents approved by the Food and Drug Administration. Recruiters at each cancer center were trained in how to approach patients and introduce the study. Eligible patients were cognitively intact; had a Karnofsky score of 50 or higher or an ECOG score of 0 to 2; had a landline or cellular telephone; were able to speak and read English; and had appropriate level of hearing for telephone contacts. If patients agreed to participate, they signed the consent form and received a folder with complete study information including who to call with questions. Recruiters entered all patient information and drug name and dosage into a secure electronic tracking system, and interviewers conducted baseline interviews shortly after patients had received their medication from the specialty pharmacy and started taking it. Following the intake interview, patients were randomized to either the experimental or control arm. A minimization program was used to balance treatment arms by accrual location, cancer site, continuous vs intermittent dosage, concurrent intravenous chemotherapy, and depression. All patients received interviews at 4, 8, and 12 weeks and weekly automated calls to assess symptoms and adherence to their oral oncolytic agents. Patients randomized to the experimental arm received daily telephone reminders to take their medications and a printed copy of the Medication Management and Symptom Management Toolkit (toolkit). During weekly calls, patients in the experimental arm were advised to consult the toolkit for each symptom they scored at a 4 or higher in severity on a 0 to 10 scale. This toolkit described 18 common symptoms associated with cancer and treatment using oral anticancer agents. It was organized by frequently asked questions and presented evidence-based advice on how to manage the symptom and when (at what severity levels) to inform the oncologist. The toolkit also offered suggestions on managing oral anticancer medication and enhancing adherence.

\section{2 | Measures}

All measures were administered at intake, 4, 8, and 12 weeks, with the exception of demographics and comorbid conditions, which were assessed at intake only. If oral cancer medication was stopped, and the patient did not take the medication in any of the 4-week period between interviews, medication-related instruments including the $\mathrm{BMQ}$ were not administered.

\subsection{1 | Beliefs about medicines (BMQ)}

Originally, the instrument contained 10 items, $^{3}$ each rated on a 1-to-5 (strongly agree to strongly disagree) scale. Five items loaded onto the necessity subscale and 5 onto the concerns subscale. In 2004, the 11th item was added, focusing on unpleasant side effects. ${ }^{19}$ For this research, 11 items were included, each with reference to "oral cancer medications." All items were submitted to an exploratory factor analysis at each of the 4 observations. Five necessity items were loaded consistently on the necessity subscale at each observation. However, at each observation, the original concern items separated into 2 unique subscales, one of which was labeled the concerns subscale, and the other one was labeled interference subscale. The concerns subscale included worry about having to take medications, worry about longterm effects, "medications are a mystery to me," and worry about becoming too dependent. The interference subscale comprised of 1 item from the original concerns subscale ("my medications disrupt my life") and 1 item from the later version of the instrument ("my medications give me unpleasant side effects"). Factor loadings for these 3 subscales remained stable at each of the 4 time points. Therefore, 3 subscale scores-necessity, concerns, and interference-were computed at each observation by averaging the corresponding item scores. Reverse scoring of items assured that higher scores on each subscale indicated greater necessity, concerns, or interference. The internal consistency reliability (Cronbach alphas) for each subscale at 4 time 


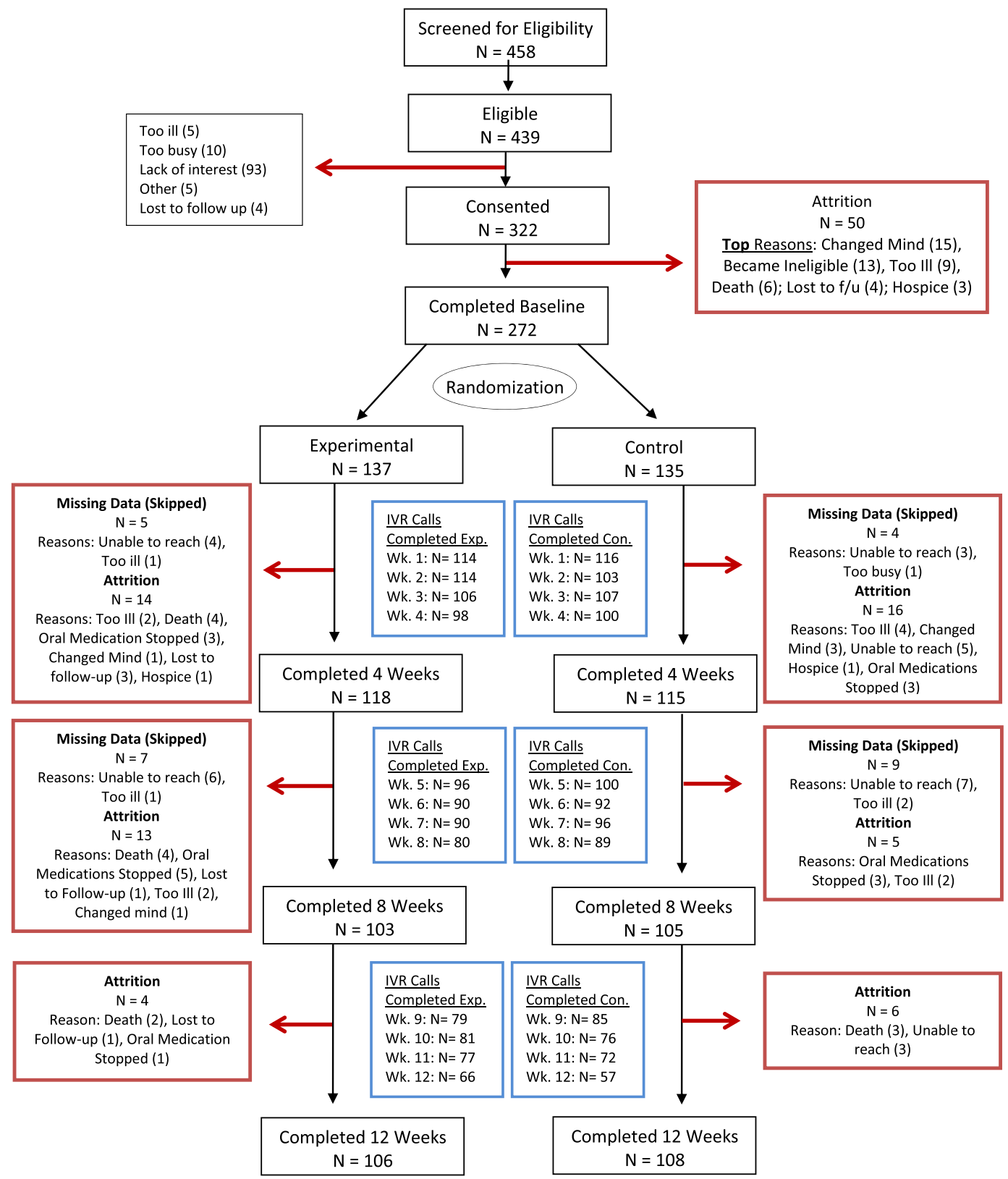

FIGURE 1 Oral adherence consort table. IVR, interactive voice response

points was $.76, .81, .83$, and .87 for necessity; .64, .68, .66, and .67 for concerns; and .75, .80, .70, and .75 for interference.

\subsection{2 | Symptoms}

The presence, severity, and interference with daily activities were measured for 18 prevalent symptoms including pain fatigue, sleep disturbance, anxiety, weakness, headaches, skin rash, numbness or tingling, redness or peeling in hands or feet, swelling of hands and feet, joint/muscle pain, mouth sores, lack of appetite, nausea or vomiting, diarrhea, constipation, cough, and shortness of breath. Patients indicated if they had experienced the symptom in the past 7 days and, if yes, rated severity of the symptom on a scale from 1 to 9 and interference of that symptom with daily activities on a scale of 0 (did not interfere) to 9 (interfered completely). Summed symptom severity and summed symptom interference indices were derived across the array of symptoms.

\subsection{3 | Depressive symptoms}

Depressive symptoms were assessed using the Center for Epidemiologic Studies Depression (CES-D) 20-item scale. ${ }^{20}$ Cronbach alpha exceeded .90 in this sample.

\subsection{4 | Physical function}

Physical function was measured ${ }^{21,22}$ using the Patient Reported Outcomes Measurement Information System Short Form 10 (Cronbach alpha exceeded .90).

\subsection{5 | Demographic characteristics}

Demographic characteristics including age, sex, race, ethnicity, and level of education were obtained during baseline interview. Level of education was summarized as high school or less, some to 4-year college education, or graduate/professional degree. 


\subsubsection{Comorbid conditions treated with medications}

Comorbid conditions treated with medications at intake into the trial were identified via electronic medical record (EMR) audits. The medications documented in the EMR prior to the prescription date for the oral oncolytic medication were used to identify the corresponding comorbid conditions. Based on the purpose of the drug, conditions included cardiovascular problems, chronic lung disease, asthma, kidney disease, diabetes, depression, and arthritis. If multiple medications were prescribed for the same condition, the condition was counted once.

\subsection{7 | Oral oncolytic agents}

Oral oncolytic agents were collapsed into 4 categories: cytotoxic agents, kinase inhibitors, sex hormone inhibitors, and others (see Appendix A). Dosages of these oral oncolytic medications were either continuous (taken every day) or intermittent (medication taken followed by rest periods).

\subsection{8 | Treatment stoppages}

Treatment stoppages were based on start date as documented in the EMR and then assigned to a corresponding 4-week time: baseline to weeks 4,4 to 8 , or 8 to 12 . Temporary stoppages were differentiated from permanent stoppages based on information in the EMR for each oral oncolytic agent. Patients could experience multiple temporary stoppages but only 1 permanent stoppage. Temporary or permanent stoppage during each 4-week period was defined by at least one of the drugs in the protocol being stopped temporarily or permanently, respectively, in that period. We were unable to identify dose reductions in a uniform manner across sample for 2 reasons. First, reductions had to be calculated for each drug among multiple ones taken by a patient. Second, many oncolytic agents had intermittent dosing ( 3 weeks on 1 week off), and dose reductions could occur via directing the patient to take a smaller dose or by extending or adding rest periods.

\section{3 | STATISTICAL ANALYSIS}

The distributions of the BMQ subscale scores and other variables were summarized with descriptive statistics. Correlations among the BMQ subscales were computed at each time point. Longitudinal mixed-effects models were used to relate 4 repeated measures (baseline, 4, 8, and 12 weeks) of each of the $3 \mathrm{BMQ}$ subscale scores to the following fixed explanatory variables: patient's age, sex, drug category, level of education, number of comorbid conditions treated with medications at intake, and trial arm. Since the intervention was not directed at beliefs about medications, we did not expect differences by trial arm but, nevertheless, adjusted for it in this secondary analysis. Time was entered as a categorical variable with levels-intake: 4,8 , and 12 weeks-to model potentially nonlinear patterns in the BMQ scores over time. Summed symptom severity as calculated at baseline 4,8 , and 12 weeks was entered as a time-varying covariate. Next, the presence or absence of permanent or temporary stoppages in each observation period (1 stoppage variable at a time) was added as a time-varying covariate.
Finally, we explored adding the physical function and CES-D scores as additional time-varying factors to gauge their effects on the $\mathrm{BMQ}$ over and above symptom severity. Least square means of the BMQ subscale scores according to time and drug category were output from the mixed models, and differences among them were tested. All statistical tests were 2-sided. The analyses were conducted in SAS 9.4.

\section{4 | RESULTS}

The sample had equal representation of males and females, and a mean age of 61 years (Table 1). At intake, health insurance covered oral oncolytic medication for $89 \%$ of patients. Fifty three percent of patients had out-of-pocket cost at intake (median $\$ 25$, range \$1-\$8300). Cytotoxic agents and kinase inhibitors accounted for most 28 oral agents prescribed at intake. Of 27 patients on sex hormone inhibitors, 26 were men with prostate cancer over 60 years of age. The average number of comorbid conditions treated with medications at intake was 3.38 , with an average of 12 medications prescribed to patients for those conditions (Table 1).

Based on the unadjusted means of the BMQ subscales (Table 1), patients' beliefs about the necessity and interference of their oral anticancer medications rise over the 12 weeks while concerns decline. Each subscale appears to assess a unique construct. Necessity did not correlate with either concerns or interference, with Pearson correlation coefficients below 0.20 at each observation. Correlations between interference and concerns were 0.36 at intake, 0.28 at week $4,0.31$ at week 8 , and 0.43 at week 12 .

The highest rate of temporary stoppages of the oral agents was in the first 4 weeks of treatment (22\%) then declined for the subsequent two 4-week periods. In contrast, permanent stoppages of oral agents were fairly stable with a slight decline from $11 \%$ in the first 4 weeks to $8 \%$ during weeks 8 to 12 (Table 1 ).

The multivariable longitudinal model for the necessity scores revealed that they were significantly higher at week 12 compared to intake, with the adjusted mean difference of 0.11 , standard error of 0.05 , and $P=.02$ (first panel of Table 2). Level of education was a significant predictor of perceived necessity; those with some to completed college had lower necessity scores than those with graduate or professional degrees or high school or less education. Patients on cytotoxic agents reported lowest necessity scores compared to other drug classes. Permanent stoppages of oral agents had a strong negative effect on necessity beliefs, with significant reductions in perceived necessity following oral agent stoppages (mean reduction of 0.50 , standard error $=0.10, P<.01$, Table 2). Age, sex, number of comorbid conditions treated with medications, trial arm, and symptom severity did not affect necessity over and above education, time, drug category, and permanent stoppages.

Concerns declined significantly over the first 4 weeks following oral agent initiation then remained stable over time (Figure 2 and Table 2). Male patients, those less educated, and those who reported greater symptom severity and temporary stoppages were more concerned about the impact of their medications. In contrast, patients 
TABLE 1 Descriptive statistics for the study sample

\begin{tabular}{|c|c|}
\hline Characteristic & $\mathrm{N}(\%)$ \\
\hline \multicolumn{2}{|l|}{ Intake $(\mathrm{N}=272)$} \\
\hline \multicolumn{2}{|l|}{ Sex } \\
\hline Male & $136(50 \%)$ \\
\hline Female & $136(50 \%)$ \\
\hline \multicolumn{2}{|l|}{ Race } \\
\hline African American & $22(8 \%)$ \\
\hline Caucasian & 241 (89\%) \\
\hline Other/unknown & $9(3 \%)$ \\
\hline \multicolumn{2}{|l|}{ Ethnicity } \\
\hline Hispanic or Latino & $5(2 \%)$ \\
\hline Not Hispanic or Latino & $260(95 \%)$ \\
\hline Unknown & $7(3 \%)$ \\
\hline \multicolumn{2}{|l|}{ Level of education } \\
\hline High school or less & $71(26 \%)$ \\
\hline Some college or completed college & $150(55 \%)$ \\
\hline Graduate or professional degree & 49 (18\%) \\
\hline Unknown & $2(1 \%)$ \\
\hline \multicolumn{2}{|c|}{ Health insurance coverage for oral oncolytic medication } \\
\hline Yes & 241 (89\%) \\
\hline No & $18(6 \%)$ \\
\hline Don't know & $13(5 \%)$ \\
\hline \multicolumn{2}{|l|}{ Drug category } \\
\hline Cytotoxic agents & 95 (35\%) \\
\hline Kinase inhibitors & $127(47 \%)$ \\
\hline Sex hormone inhibitors & $27(10 \%)$ \\
\hline Other & $23(8 \%)$ \\
\hline \multicolumn{2}{|l|}{ Site of cancer } \\
\hline Breast & 57 (21\%) \\
\hline Colorectal & 41 (15\%) \\
\hline GI & $17(6 \%)$ \\
\hline Leukemia & $16(6 \%)$ \\
\hline Liver & $12(4 \%)$ \\
\hline Lung & $10(4 \%)$ \\
\hline Lymphoma & $3(1 \%)$ \\
\hline Melanoma & $8(3 \%)$ \\
\hline Myeloma & $7(3 \%)$ \\
\hline Pancreatic & $27(10 \%)$ \\
\hline Prostate & 26 (10\%) \\
\hline Renal & 24 (9\%) \\
\hline Sarcoma & 15 (5\%) \\
\hline Brain & $2(1 \%)$ \\
\hline Esophageal & $3(1 \%)$ \\
\hline Other & $4(1 \%)$ \\
\hline \multicolumn{2}{|l|}{ Study group } \\
\hline Experimental & 137 (50\%) \\
\hline Control & 135 (50\%) \\
\hline Characteristic & Mean (StDev) \\
\hline Age & $61.38(12.22)$ \\
\hline $\begin{array}{l}\text { Number of comorbid conditions treated with } \\
\text { medications }\end{array}$ & $3.38(1.99)$ \\
\hline
\end{tabular}

(Continues)
TABLE 1 (Continued)

\begin{tabular}{|c|c|}
\hline Characteristic & Mean (StDev) \\
\hline $\begin{array}{l}\text { Number of medications other than oral oncolytics } \\
\text { prescribed at intake or during the study }\end{array}$ & $11.81(5.90)$ \\
\hline Out-of-pocket cost for oral oncolytic medication & $257.82(925.53)$ \\
\hline BMQ necessity & $3.70(0.70)$ \\
\hline BMQ concerns & $2.66(0.78)$ \\
\hline BMQ interference & $2.50(0.97)$ \\
\hline Symptom severity & $24.76(22.00)$ \\
\hline Physical function & $45.20(7.87)$ \\
\hline CES-D & $9.76(8.97)$ \\
\hline \multicolumn{2}{|c|}{ Week 4 ( $N=233$ completed interview, $N=222$ completed BMQ) } \\
\hline BMQ necessity & $3.62(0.77)$ \\
\hline BMQ concerns & $2.51(0.76)$ \\
\hline BMQ interference & $2.67(1.1)$ \\
\hline Symptom severity & $22.61(21.43)$ \\
\hline Physical function & $44.94(8.39)$ \\
\hline CES-D & $9.69(8.75)$ \\
\hline Had a temporary drug stoppage, $\mathrm{N}(\%)^{\mathrm{a}}$ & $57(22 \%)$ \\
\hline Had a permanent drug stoppage, $\mathrm{N}(\%)^{\mathrm{a}}$ & $28(11 \%)$ \\
\hline \multicolumn{2}{|c|}{ Week 8 ( $N=208$ completed interview, $N=186$ completed BMQ) } \\
\hline BMQ necessity & $3.72(0.77)$ \\
\hline BMQ concerns & $2.47(0.77)$ \\
\hline BMQ interference & $2.63(1.00)$ \\
\hline Symptom severity & $18.82(16.18)$ \\
\hline Physical function & $45.35(7.92)$ \\
\hline CES-D & $9.15(8.21)$ \\
\hline Had a temporary drug stoppage, $\mathrm{N}(\%)^{\mathrm{a}}$ & $30(12 \%)$ \\
\hline Had a permanent drug stoppage, $N(\%)^{a}$ & $24(9 \%)$ \\
\hline \multicolumn{2}{|c|}{ Week 12 ( $N=214$ completed interview, $N=164$ completed BMQ) } \\
\hline BMQ necessity & $3.80(0.87)$ \\
\hline BMQ concerns & $2.47(0.77)$ \\
\hline BMQ interference & $2.62(1.04)$ \\
\hline Symptom severity & $17.99(17.58)$ \\
\hline Physical function & $45.58(8.45)$ \\
\hline CES-D & $9.52(8.83)$ \\
\hline Had a temporary drug stoppage, $\mathrm{N}(\%)^{\mathrm{a}}$ & $12(5 \%)$ \\
\hline Had a permanent drug stoppage, $\mathrm{N}(\%)^{\mathrm{a}}$ & $18(8 \%)$ \\
\hline
\end{tabular}

Abbreviations: BMQ, Beliefs About Medications Questionnaire; CES-D, Center for Epidemiologic Studies Depression.

apercent is out of the number of patients with available medical record audit data for each period: $N=272$ at intake, $N=265$ at week 4 , $\mathrm{N}=255$ at week 8 , and $\mathrm{N}=234$ at week 12 .

whose treatment was not interrupted had fewer concerns regarding the impact of their medications.

Interference scores increased significantly between baseline and week 4 then were relatively stable between weeks 4 and 12 (Figure 2 and Table 2). Higher symptom severity and male sex were associated with greater interference.

When physical function and CES-D scores were added to the longitudinal models as time-varying covariates, they were not significant and did not appreciably change the effects of other variables, therefore were not included in the final models. 
TABLE 2 Longitudinal models for BMQ subscales: coefficients, SEs, and significance of the effects of the explanatory variables

\begin{tabular}{|c|c|c|c|c|c|c|c|c|c|}
\hline \multirow[b]{2}{*}{ Explanatory Variable } & \multicolumn{3}{|l|}{ Necessity } & \multicolumn{3}{|l|}{ Concerns } & \multicolumn{3}{|l|}{ Interference } \\
\hline & Coefficient (SE) & $\mathrm{T}$ & $P$ & Coefficient (SE) & $T$ & $P$ & Coefficient (SE) & $T$ & $P$ \\
\hline Age & $-0.0027(0.28)$ & -0.80 & 0.42 & $0.0011(0.003)$ & 0.33 & .74 & $0.0009(0.004)$ & 0.23 & .82 \\
\hline \multicolumn{10}{|l|}{ Sex } \\
\hline Male & $-0.13(0.08)$ & -1.60 & 0.11 & $0.20(0.08)$ & 2.57 & .01 & $0.17(0.09)$ & 1.95 & .05 \\
\hline \multicolumn{10}{|l|}{ Trial arm } \\
\hline Experimental & $0.09(0.08)$ & 1.21 & 0.23 & $-0.05(0.07)$ & -0.72 & .47 & $-0.05(0.08)$ & -0.58 & .56 \\
\hline \multicolumn{10}{|l|}{ Control (ref) } \\
\hline Symptom severity & $0.0005(0.001)$ & 0.35 & 0.73 & $0.006(0.0013)$ & 4.54 & $<.01$ & $0.02(0.002)$ & 13.24 & $<.01$ \\
\hline \multicolumn{10}{|l|}{ Time } \\
\hline \multicolumn{10}{|l|}{ Intake (ref) } \\
\hline \multicolumn{10}{|l|}{ Drug category } \\
\hline Cytotoxic agents & $-0.25(0.14)$ & -1.73 & 0.08 & $-0.14(0.14)$ & -0.99 & .32 & $0.55(0.15)$ & 3.61 & $<.01$ \\
\hline Kinase inhibitors & $-0.07(0.14)$ & -0.48 & 0.60 & $0.07(0.14)$ & 0.54 & .60 & $0.45(0.15)$ & 3.01 & $<.01$ \\
\hline Other & $0.01(0.18)$ & 2.34 & 0.94 & $-0.11(0.18)$ & -0.62 & .54 & $0.51(0.19)$ & 2.61 & .01 \\
\hline \multicolumn{10}{|l|}{$\begin{array}{l}\text { Sex hormone inhibitors } \\
\text { (ref) }\end{array}$} \\
\hline \multicolumn{10}{|l|}{ Oral agent stoppage } \\
\hline Yes & Permanent: $-0.50(0.10)$ & -5.18 & $<0.01$ & Temporary: 0.15 (0.06) & 2.54 & .01 & Permanent: 0.20 (0.14) & 1.46 & .16 \\
\hline \multicolumn{10}{|l|}{ No (ref) } \\
\hline
\end{tabular}

Abbreviations: BMQ, Beliefs About Medications Questionnaire; SE, standard error.

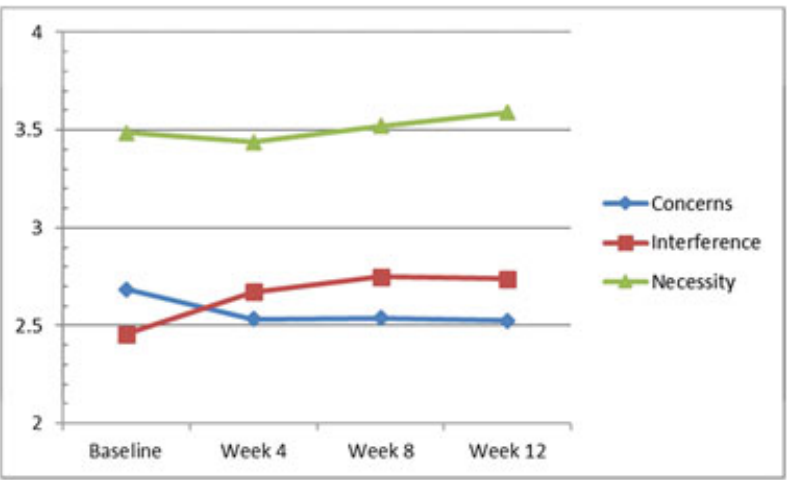

FIGURE 2 Least square means for the Beliefs About Medications Questionnaire necessity, concerns, and interference at 4 time points

\section{5 | DISCUSSION}

A new generation of oral oncolytic agents is transforming cancer treatment. At least a quarter (1/4) of all cancer agents in development are oral agents. Therefore, it is important to understand how events arising during treatment with oral oncolytic agents affect patients' beliefs regarding the necessity, concerns, and interference they attribute to these medications. Consistent with other findings, ${ }^{23}$ the 3 subscales have low to moderate interscale correlations, indicating that they reflect distinct constructs that change differently over time.

Patients' beliefs in the necessity of their medications did not change in the first 4 weeks then increased significantly between weeks 4 and 12 . Concerns about medications declined in the first 4 weeks, with no change in weeks 8 to 12, and beliefs about medication interference steadily increased over time. With respect to necessity, the initial 4 weeks following the initiation of treatment appeared to be a time for deliberation. Following this initial period, patients altered their beliefs (stronger beliefs in the necessity of their medications, except for those where the medications were stopped; fewer concerns about its impact on future health; and a realization that the medications interfere with daily activities). However, changes in these beliefs are grounded in the events surrounding treatment. As symptom severity worsens, patients report greater concerns about how medications may affect their future health. Increasing symptom severity does not appear to affect beliefs about 
necessity and may indicate that the treatment is working, and thus, medication is necessary to manage the cancer.

This research is among the first to show how patients' beliefs in the necessity of their medications decline when medications are withdrawn by the physician. Consistent with several cross-sectional studies of beliefs about different medication types, ${ }^{16,24}$ we found that treatment interruptions may give rise to concerns that oral oncolytic agents are no longer managing their disease. At least 2 lines of explanation might be pursued. First, patients' beliefs regarding a medication may be mediated by oncologists' views of its efficacy. This explanation ${ }^{25}$ points to the pivotal role that oncologists play in defining the necessity of treatment. Second, once stopped, patients may disavow the necessity of their medication as a form of reducing cognitive dissonance. While speculative, these explanations may guide future research regarding abrupt shift in necessity once cancer treatments are stopped.

Patients' concerns prompted by treatment interruption may produce a different type of reaction than concerns resulting from worsening symptoms. Interruptions suggest to patients that the medication is not tolerable while increasing symptoms may engender mixed reactions; on one hand, symptoms may indicate that the drug is working but concerns may be rising because patients may be unable to endure the consequences of the medication. Similar lines of research using other chronic diseases have reported how lack of self-efficacy was related to beliefs about necessity and concerns. ${ }^{13,26}$ These data indicate that concerns regarding oral agents arise from different sources. Future research needs to explore how patients interpret and act upon concerns depending upon the factors that gave rise to them.

Regarding the effects of other factors on beliefs, differences according to sex and level of education were found, but comorbidity did not affect beliefs over and above other factors in longitudinal models. These findings are in contrast with those in type 2 diabetes, where differences in necessity beliefs by sex and comorbid conditions were found among patients on antihypertensive medications. ${ }^{26}$ Lack of differences by sex and comorbidity in this study could be because of the difference in populations and the fact that oral oncolytic medications are often prescribed as the last available line of treatment. On the other hand, our findings on the influence of education on beliefs are similar to those reported among people with asthma. ${ }^{27}$

Finally, there were no effects of the adherence reminder and symptom management intervention on beliefs over and above symptom severity. This finding was expected because the intervention was not directed toward altering beliefs.

\section{6 | CONCLUSIONS}

In summary, patients' beliefs about the necessity of their cancer treatments are high, increase over time, and decline only when medications are permanently stopped. Male patients, those who report increasing severity of their symptoms, and those who experience temporary stoppages of their medications report increased concerns and interference of their medications with their lives. Since these factors remain significant in the multivariable model, they represent relatively independent contributions toward patients' level of concern.

\section{1 | Limitations}

The small numbers of racial and ethnic minorities limited assessment of how these variables affected beliefs. The follow-up period was very short given the long-term treatment among patients on these medications. Finally, during this short period, adherence was high and had little variation, which precluded the examination of the effects of beliefs on adherence.

\section{2 | Clinical implications}

Oncologists could be made aware of how medication adjustment occurs over time, introduce supportive and end-of-life care, and present it as a valuable next line of cancer management. Providers of supportive and end-of-life care could address patient beliefs about oral oncolytic medications as part of comprehensive patient care as patients move toward the end of life.

\section{ACKNOWLEDGEMENT}

This work was supported by the National Cancer Institute (grant number 1R01CA162401-01A1. 2013-2017).

\section{ORCID}

Barbara A. Given (1D http://orcid.org/0000-0002-3971-214X

\section{REFERENCES}

1. Weingart SN, Brown E, Bach PB, et al. NCCN task force report: oral chemotherapy. J Natl Compr Canc Netw. 2008;6(3):S1-S16.

2. Horne R, Weinman J. Patients' beliefs about prescribed medicines and their role in adherence to treatment in chronic physical illness. $J$ Psychosom Res. 1999;47(6):555-567.

3. Horne R, Weinman J, Hankins M. The beliefs about medicines questionnaire: the development and evaluation of a new method for assessing the cognitive representation of medication. Psychol Health. 1999;14(1):1-24.

4. Becker $\mathrm{MH}$. The health belief model and sick role behavior. Health Educ Quart. 1974;2:409-419.

5. Janz NK, Becker MH. The health belief model: a decade later. Health Educ Behav. 1984;11(1):1-47.

6. Rosenstock IM. Historical origins of the health belief model. Health Educ Quart. 1974;2:328-335.

7. Horne R, Chapman SCE, Parham R, Freemantle N, Forbes A, Cooper V. Understanding patients' adherence-related beliefs about medicines prescribed for long-term conditions: a meta-analytic review of the necessity-concerns framework. PLoS One. 2013;8(12):e80633

8. Andersson Sundell K, Jonsson AK. Beliefs about medicines are strongly associated with medicine-use patterns among the general population. Int J Clin Pract. 2016;70(3):277-285.

9. Kumar K, Raza K, Nightingale P, et al. Determinants of adherence to disease modifying anti-rheumatic drugs in white British and South Asian patients with rheumatoid arthritis: a cross sectional study. BMC Musculoskelet Disord. 2015;16(1):396

10. Bhattacharya D, Easthall C, Willoughby KA, Small M, Watson S. Capecitabine non-adherence: exploration of magnitude, nature and contributing factors. J Oncol Pharm Pract. 2012;18(3):333-342.

11. Timmers L, Boons CC, Mangnus D, et al. Adherence and patients' experiences with the use of capecitabine in daily practice. Front Pharmacol. 2016;7:article 310 
12. De las Cuevas C, de Leon J. Reviving research on medication attitudes for improving pharmacotherapy: focusing on adherence. Psychother Psychosom. 2017;86(2):73-79.

13. Devlen J, Beusterien K, Yen L, Ahmed A, Cheifetz AS, Moss AC. Barriers to mesalamine adherence in patients with inflammatory bowel disease: a qualitative analysis. J Manag Care Spec Pharm. 2014;20(3):309-314.

14. Michetti $P$, Weinman J, Mrowietz $U$, et al. Impact of treatment-related beliefs on medication adherence in immune-mediated inflammatory diseases: results of the global ALIGN study. Adv Ther. 2017;34(1):91108.

15. Náfrádi L, Galimberti E, Nakamoto K, Schulz PJ. Intentional and unintentional medication non-adherence in hypertension: the role of health literacy, empowerment and medication beliefs. J Public Health Res. 2016;5(3):762

16. Salgado TM, Davis EJ, Farris KB, Fawaz S, Batra P, Henry NL. Identifying socio-demographic and clinical characteristics associated with medication beliefs about aromatase inhibitors among postmenopausal women with breast cancer. Breast Cancer Res Treat. 2017;163(2):311319.

17. Timmers L, Boons CC, Kropff F, et al. Adherence and patients' experiences with the use of oral anticancer agents. Acta Oncol. 2014;53(2):259-267.

18. Given BA, Given CW. Improving adherence to oral cancer agents and self care of symptoms using an IVR. National Cancer Intstitute grant number 1R01CA162401-01A1. 2013-2017

19. Horne R, Buick D, Fisher M, Leake H, Cooper V, Weinman J. Doubts about necessity and concerns about adverse effects: identifying the types of beliefs that are associated with non-adherence to HAART. Int J STD AIDS. 2004 Jan;15(1):38-44.

20. Radloff LS. The CES-D Scale: a self-report depression scale for research in the general population. Appl Psychol Measur. 1977;1(3):385-401.

21. Cella D, Young S, Rothrock N, et al. The patient-reported outcomes measurement information system (PROMIS): progress of an $\mathrm{NIH}$ roadmap cooperative group during its first two years. Med Care. 2007;45(5 Suppl):S3-S11.

22. PROMIS. Adult physical function profile short forms. 2013. http:// assessmentcenter.net Accessed May 1, 2017.

23. Foot H, La Caze A, Gujral G, Cottrell N. The necessity-concerns framework predicts adherence to medication in multiple illness conditions: a meta-analysis. Patient Educ Couns. 2016;99(5):706-717.

24. De Smedt RH, Denig P, van der Meer K, Haaijer-Ruskamp FM, Jaarsma T. Self-reported adverse drug events and the role of illness perception and medication beliefs in ambulatory heart failure patients: a cross-sectional survey. Int J Nurs Stud. 2011;48(12):1540-1550.

25. Grassi L, Meggiolaro E, Berardi MA, et al. Beliefs about medicines, doctor-patient relationship, and coping among European patients with cancer. Psycho-Oncol. 2017;26(2):282-285.

26. Aikens JE, Piette JD. Diabetic patients' medication underuse, illness outcomes, and beliefs about antihyperglycemic and antihypertensive treatments. Diabetes Care. 2009;32(1):19-24.

27. Federman AD, Wolf $M$, Sofianou A, et al. The association of health literacy with illness and medication beliefs among older adults with asthma. Patient Educ Couns. 2013;92(2):273-278.

How to cite this article: Sikorskii A, Given CW, Given BA, et al. Do treatment patterns alter beliefs cancer patients hold regarding oral oncolytic agents? Psycho-Oncology. 2018;27: 1005-1012. https://doi.org/10.1002/pon.4606

\section{APPENDIX}

\section{ORAL AGENT DRUG CATEGORIES}

\begin{tabular}{lc} 
Cytotoxics & \\
\hline Temodar (temozolomide) & $7(2.6)$ \\
Lonsurf (tipiracil and trifluridine) & $1(0.3)$ \\
Xeloda (capecitabine) & $92(33.8)$ \\
Kinase inhibitors & \\
\hline
\end{tabular}

$B R C-A B L$ tyrosine kinase inhibitor

Bosulif (bosutinib)

Gleevec (imatinib)

Sprycel (dasatinib)

Tasigna (nilotinib)

VEGF/VEGFR inhibitor

Inlyta (Axitinib)

Nexavar (Sorafenib)

$11(4.0)$

Sutent (Sunitinib)

8 (2.9)

Votrient (Pazopanib)

$22(8.1)$

Lenvima (Lenvatinib)

$1(0.3)$

Stivarga (Regorafenib)

9 (3.3)

EGFR HER2/neu

Tarceva (erlotinib)

5 (1.8)

Gilotrif (afatinib)

$1(0.3)$

Tykerb (lapatinib)

$1(0.3)$

ALK inhibitor

Xalkori (crizotinib)

Zykadia (ceritinib)

BRAF inhibitor

Tafinlar (dabrafenib)

Phospoinositide 3-kinase inhibitor

Zydelig (idelalisib)

CDK inhibitor

Ibrance (palbociclib)

Bruton tyrosine kinase inhibitor

Imbruvica (ibrutinib)

Sex hormone inhibitors

Xtandi (enzalutamide)

$18(6.6)$

Zytiga (abiraterone acetate)

Other

IMIDS

Revlimid (lenalidomide)

Pomalyst (pomalidomide)

mTOR inhibitors

Afinitor (everolimus)

PARP inhibitor

Lynparza (olaparib)

Abbreviations: ALK, anaplastic lymphoma kinase; CDK, cyclin dependent kinase; EGFR, epidermal growth factor receptor; IMIDS, immunomodulatory; mTOR, mechanistic target of rapamycin; PARP, poly adenosine diphosphate ribose polymerase; VEGR, vascular endothelial growth factor; VEGFR, vascular endothelial growth factor receptor. 